\title{
The Clinical Use of Mushrooms in Oncology
}

\author{
Steven Kh Aung
}

9904-106 Street NW, Edmonton, Alberta T5K 1C4, Canada, email: draung@aung.com, website: http://www.aung.com

Oncology, from a traditional Chinese medicine (TCM) perspective, is known as Ai Zhi, which signifies physiological accumulation of the "mass," which is pushing out and spreading to various cells and organs in the body. It is also associated with the accumulation of phlegm, which may be generally defined as a pathogenic, chronic accumulation of mucus in various organ systems, especially the stomach and the bladder organ/meridian systems, which causes severe blockage of Qi (vital energy), subsequently causing blood stagnation and mass formation. This may lead to cancer. Another major cause of cancer, according to TCM theory, is blood stasis. In TCM oncology, the major emphasis is to eliminate phlegm and blood stagnation/stasis. Various methods are used for these purposes, and nutritional/herbal medicine plays a central role. Mushrooms were proven clinically useful in fighting cancer, especially the Ganoderma lucidum (W.Curt.:Fr.)Lloyd (Ling Zhi or Reishi), Lentinus edodes (Berk.)Singer (Shiitake) species.

Mushrooms are good not only for immunoenhancement, but also to complement Western chemotherapy and radiation therapy. Mushrooms contain a number of polysaccharides and secondary metabolites, which work by countering the side-effects of cancer such as nausea, bone marrow suppression, anemia, and lowered resistance.

It is also vitally important to consider the aspects of internal body harmony in conjunction with the mental and spiritual aspects, involving the suffering patients, their families and friends, and the various physicians and other caregivers involved. In medical acupuncture clinics, certain medicinal mushroom products approved by the Canadian government and other governments are useful adjuncts in treating invasive tumors and the pain of cancer as well as the side-effects of chemotherapy, radiation, and surgery, such as the following:

- Comely: this capsule, made in Hong Kong, contains wild Ling Zhi (with several of its polysaccharides and secondary metabolites) as well as various pollens and also saponins of $G y$ nostemma pentaphylla (a species of Ginseng).

- Golden Lu Bao Ling Zhi: this capsule, made in Hong Kong, is an extract of Ling Zhi, containing six different wild Ling Zhi species gathered from the mountains of the Taishan region of China.

- Ginseng, Reishi Pearl, and Pollen Tablet: made in Guangzhou, China, the main ingredients of this tablet are Ginseng (30\%), wild Ling Zhi (25\%), and honey enriched pollen (25\%).

- Yun Zhi: a capsule made in Guiyang, China, it is 100\% Trametes versicolor (L.:Fr.)Pilát (=Coriolus versicolor) (Lu Bao Yun Zhi mushroom).

The above products containing various mushroom ingredients are all sterile, purified, carefully packaged, quality-controlled products approved by the Canadian government for appropriate medical distribution. In my own medical acupuncture clinic, they are prescribed as adjunctive methods.

In the traditional Chinese medical system, acupuncture is thought to have a relatively fast effect on pain control and vital energy (Qi) balancing, whereas nutritional and herbal therapeutic modalities have a slower but deeper effect in the major internal organs. From an integrative medical perspective, cancer and other patients are required to maintain close and direct contact with their own personal family physicians and specialists, such as oncologists, for optimum health for all concerned. 